\title{
Seminal Vesicle Cystadenoma
}

National Cancer Institute

\section{Source}

National Cancer Institute. Seminal Vesicle Cystadenoma. NCI Thesaurus. Code C39907.

A rare benign cystadenoma that arises from the seminal vesicle. 